\title{
Iterative model and trajectory refinement for orbital trajectory optimization
}

\author{
Jennifer Hudson ${ }^{1}$ (1) | Rohit Gupta ${ }^{2}$ | Nan Li $^{2}$ | Ilya Kolmanovsky²
}

\begin{abstract}
${ }^{1}$ Department of Mechanical and Aerospace Engineering, Western Michigan University, Kalamazoo, MI 49008-5343, USA

${ }^{2}$ Department of Aerospace Engineering, University of Michigan, Ann Arbor, MI 48109-2140, USA
\end{abstract}

\section{Correspondence}

Jennifer Hudson, Department of Mechanical and Aerospace Engineering, Western Michigan University, Kalamazoo, MI 49008-5343, USA.

Email: jennifer.hudson@wmich.edu

Funding information

National Science Foundation Award, Grant/Award Number: CNS 1544844

\begin{abstract}
Summary
An iterative model and trajectory refinement (IMTR) strategy is proposed for trajectory optimization of nonlinear systems. A high- and a low-fidelity models are used. The high-fidelity model accurately represents the system but is not easily amenable to trajectory optimization, because of degree of nonlinearity, computational cost, or to being of "black-box" type. The low-fidelity model is suitable for numerical optimization but approximates the system dynamics with an error. The IMTR method is proposed to systematically iterate between the 2 models and efficiently converge on a control solution. Examples are drawn from orbital mechanics. The IMTR approach is compared to optimal nonlinear quadratic control using Pontryagin maximum principle. A convergence criterion for the IMTR iterations is established.
\end{abstract}

\section{KEYWORDS}

iterative/numerical methods, optimal control, orbital trajectory optimization

\section{1 | INTRODUCTION}

It is common in many applications for a system to be described by multiple models of varying fidelity and complexity. Often a high-fidelity model accurately represents the system dynamics but is computationally complex and requires long simulation time. These conditions can make trajectory optimization difficult. Numerical optimization methods require many iterations, and, in the absence of a good initial guess, they may not converge.

A low-fidelity model of the system may be more amenable to trajectory optimization. Such a model requires a shorter simulation time and can be optimized by analytical methods (eg, LQ optimal control, if the low-fidelity model is linear and the cost is quadratic). However, the low-fidelity model does not accurately represent the full system dynamics, and an optimal control derived from the low-fidelity model alone may not achieve the desired system output.

Iterative model and trajectory refinement (IMTR) is a recently proposed trajectory optimization strategy ${ }^{1,2}$ that systematically uses both a high- and a low-fidelity model of a system. The method uses intertwined steps of trajectory optimization on the low-fidelity model and disturbance estimation to make the low-fidelity model locally match the high-fidelity model. Compared to numerical optimization of the high-fidelity model alone, fewer simulations of the high-fidelity model may be required, which reduces computational costs.

This paper considers the application of IMTR to two spacecraft-maneuvering problems in which spacecraft dynamics are represented by both low- and high-fidelity models: an orbital transfer problem and a problem of optimal control near libration points in the 3-body problem. The orbital transfer problem can be described by nonlinear equations of 2-body orbital motion, or the dynamics can be approximated by the linear Clohessy-Wiltshire equations. ${ }^{3}$ Spacecraft dynamics near a libration point can be described by the nonlinear circular restricted 3-body model, or approximated by linearized equations for perturbation about the equilibrium.

We address the orbital transfer problem using two different approaches. The first uses nonlinear quadratic (NLQ) control 
with Pontryagin's maximum principle (hereafter, called the maximum principle) to minimize a quadratic cost function of the high-fidelity trajectory. The second approach uses IMTR to optimize the same quadratic cost function through optimization of the low-fidelity model.

Having validated IMTR in comparison with the maximum principle solution in the orbital transfer problem, we address the problem of spacecraft dynamics near a libration point using the IMTR approach only.

In both of our spacecraft examples, the dynamics are highly nonlinear for large maneuvers; the linearization-based low-fidelity model alone is not sufficiently accurate. As we demonstrate, however, such a model can lead to effective, precise, and near-optimal maneuvers once enhanced with IMTR.

A preliminary study of IMTR for spacecraft trajectory optimization has appeared in previous studies. ${ }^{4,5}$ The present paper presents a significantly more in-depth treatment of the case studies and derives theoretical conditions for IMTR iterations convergence.

This paper focuses on high-fidelity models that are nonlinear and low-fidelity models that are linear time-invariant with additive, time-dependent disturbance input. Thus, the low-fidelity models are not required to be time-varying linearizations of the system around a nominal trajectory. This approach reduces the trajectory optimization problem to a repeated solution of an LQ-type problem, where a significant portion of the solution can be precomputed offline.

There is a growing interest in solving the LQ problem for uncertain systems. ${ }^{6}$ The proposed approach is related to iterative learning control (ILC) $)^{7,8}$ and iterative dynamic programming (IDP). ${ }^{9}$ These techniques are different from IMTR: ILC primarily addresses trajectory tracking where the target trajectory is known rather than an optimal control problem, and neither ILC nor IDP exploit cost minimization based on low-fidelity and high-fidelity dynamic models as considered in IMTR. The proposed approach is also related to iterative schemes for solving optimal control problems for bilinear systems $^{10-13}$; however, IMTR is not limited to high-fidelity models of a particular form.

The paper is organized as follows. In Section 2, the IMTR algorithm is described. Section 3 analyzes IMTR iterations convergence and derives sufficient conditions under which this convergence takes place. An example for which these sufficient conditions are satisfied is provided. In Section 4, a condition is given under which the solution obtained by IMTR is guaranteed to be a near-optimal solution, ie, it deviates from the optimal solution of the high-fidelity model by less than a bound. In Section 5, the NLQ and IMTR methods are applied to an example of an orbital maneuvering optimal control problem. In Section 6, the IMTR approach is applied to an example of optimal control about the $L_{4}$ Lagrange point in the Earth-Moon system. Following the examples, concluding remarks are made in Section 7.
Trajectory optimization is an inherent part of mission planning for spacecraft applications (see, eg, Conway ${ }^{14}$ ). While it is a common practice to use different levels of models and approximations in spacecraft trajectory design (eg, patched conics followed by optimization on a higher fidelity nonlinear simulation model), this is done heuristically and differently from IMTR. In the present paper, we set up an iterative process where the low-fidelity model is tunable and matched to the higher fidelity model in each iteration, and the optimization is performed only on the low-fidelity model.

Spacecraft trajectory optimization is one of many possible areas of application for IMTR. Similar computational strategies can be exploited in other trajectory optimization problems, where the dynamic system can be represented by low-fidelity and high-fidelity models. As an example, in Hudson et al, ${ }^{1}$ IMTR strategy is applied to clutch trajectory optimization in an automotive vehicle, where the low- and high-fidelity models are of drastically different orders and complexity. In Gupta et al, ${ }^{2}$ IMTR is applied to a nonlinear engine control problem with the low-fidelity model on the basis of a linearization at a given operating point.

The numerical examples in this paper and further references ${ }^{1,2}$ indicate that the IMTR strategy can be successful in treating a variety of practical optimal control problems. The analysis in Section 3 establishes sufficient conditions for the IMTR iterations to converge.

\section{2 | THE ITERATIVE MODEL AND TRAJECTORY REFINEMENT ALGORITHM}

We consider a system described by a nonlinear, high-fidelity, state-space model,

$$
\dot{x}_{h}=f\left(x_{h}, u\right),
$$

where $x_{h}$ is a vector of state variables and $u$ is the manipulated input. The system is also approximated by a linear low-fidelity model,

$$
\dot{x}_{l}=A x_{l}+B u+d,
$$

where $x_{l}$ is a vector of state variables, $A$ and $B$ are constant matrices, and $d$ is a time-dependent disturbance input that we iteratively adjust to improve the match between Equations 1 and 2. The subscripts $l$ and $h$ designate the lowand high-fidelity models, respectively. While extensions are possible, the dimensionalities of the state and control vectors are assumed to be the same for both the low-fidelity and high-fidelity models. The initial conditions are fixed and $x_{h}(0)=x_{l}(0)$.

It is assumed that the high-fidelity model (Equation 1) accurately describes the system, while the low-fidelity model (Equation 2) describes the system with an error. Note that the linear model is not required to be a time-varying lin- 
earization about the high-fidelity model trajectory. While the low-fidelity model (Equation 2) may be defined as a linearization of the nominal model about some nominal (possibly equilibrium) state and control values, it is not required to be.

The objective is to find the control $u(t)$, defined over the time interval $t \in[0, T]$, that minimizes a quadratic cost function,

$$
J_{h}=\frac{1}{2} x_{h}(T)^{T} K_{f} x_{h}(T)+\frac{1}{2} \int_{0}^{T}\left[x_{h}(t)^{T} Q x_{h}(t)+u(t)^{T} R u(t)\right] d t,
$$

where $Q^{T}=Q \geq 0, R^{T}=R>0$, and $K_{f}^{T}=K_{f} \geq 0$.

The optimal solution to Equation 3 is not readily available, as the high-fidelity model is nonlinear. However, a similar problem for the linear low-fidelity model (Equation 2),

$$
J_{l}=\frac{1}{2} x_{l}(T)^{T} K_{f} x_{l}(T)+\frac{1}{2} \int_{0}^{T}\left[x_{l}(t)^{T} Q x_{l}(t)+u(t)^{T} R u(t)\right] d t,
$$

can be solved easily. The optimal solution to the problem of minimizing Equation 4 is given by

$$
u=-R^{-1} B^{T} P x_{l}+R^{-1} B^{T} r,
$$

where $P$ and $r$ are given by

$$
\begin{gathered}
-\dot{P}=A^{T} P+P A-P B R^{-1} B^{T} P+Q, \\
-\dot{r}=\left(A-B R^{-1} B^{T} P\right)^{T} r-P d,
\end{gathered}
$$

with $P(T)=K_{f}$ and $r(T)=0$ (see, eg, Isidori ${ }^{15}$ ). Note that Equation 6 is a Riccati equation; the solution of which can be computed independently of the iterative optimization process.

The IMTR algorithm is summarized in Table 1. Let the superscript $n$ indicate the iteration number.

The disturbance may be initialized at 0 or any other reasonable value. During each iteration, IMTR solves the low-fidelity optimization problem and estimates the disturbance $d(t)$. The disturbance update is given by

$$
d^{n+1}(t)=d^{n}(t)+k\left(\hat{\dot{x}}_{h}^{n}(t)-\hat{\dot{x}}_{l}^{n}(t)\right),
$$

where $k>0, \hat{x}_{h}^{n}(t)$ is the estimate of the time rate of change of $x_{h}$ given by the right-hand side of Equation 1 with $x_{h}=x_{h}^{n}, u=$ $u^{n}$ and $\hat{\dot{x}}_{l}^{n}(t)$ is given by Equation 2 with $u=u^{n}, d=d^{n}$. The

\section{TABLE 1 IMTR algorithm}

1. Initialize the disturbance for the first iteration, $d^{0}$.
2. Solve the finite horizon LQ problem for the low-fidelity
model to obtain $u^{n}$ and $x_{l}^{n}$.
3. Apply $u^{n}$ to the high-fidelity model to obtain $x_{h}^{n}$.
4. $\quad$ Compare $x_{l}^{n}$ and $x_{h}^{n}$. Update the disturbance.
5. $\quad$ Repeat steps 2 through 4 until the algorithm converges.

estimate of the time rate of change of $x_{h}^{n}, \hat{x}_{h}^{n}(t)$, can be obtained by evaluating the right-hand side of Equation 1 on $x_{h}^{n}$ and $u^{n}$. The estimate of the time rate of change of $x_{l}^{n}, \hat{x}_{l}^{n}(t)$, can be obtained by evaluating the right-hand side of Equation 2 on $x_{l}^{n}$, $u^{n}$, and $d^{n}$. Alternatively, standard numerical differentiation or filtering techniques can be applied to obtain $\hat{x}_{h}^{n}(t)$ and $\hat{x}_{l}^{n}(t)$.

The refinement of $d(t)$ causes the low-fidelity model to match the high-fidelity model more closely with each iteration. The constant $k$ is a gain controlling the change in the disturbance estimate with each iteration. Typically, a value of $k$ between 0 and 1 is chosen.

In principle, Equation 8 may be replaced by updating parameters in a parametric disturbance representation of the form,

$$
d(t)=\sum_{i=1}^{m} \theta_{i} \phi_{i}(t)
$$

where $\phi_{i}(t)$ are given basis functions and $\theta_{i}$ are estimated parameters.

Each iteration of the IMTR algorithm requires only one simulation of the high-fidelity model. We are never required to numerically optimize the high-fidelity model; all optimization is performed on the low-fidelity model.

Remark 1. Step 3 of the IMTR algorithm can be modified to include a check for decreasing high-fidelity model cost, $J_{h}$. After obtaining $u^{n}$ on the low-fidelity model and applying it to the high-fidelity model, if $J_{h}$ has increased, the control reverts to $u^{n-1}$. The disturbance alone is updated until the low-fidelity model reaches a form for which the optimal control also gives a decrease in $J_{h}$. This modification ensures that algorithm iterations only give a decrease in the cost and the disturbance estimate is updated multiple times between control updates. The orbital transfer problem in Section 5 uses this approach.

Remark 2. In this paper, the dimensionalities of the states of Equations 1 and 2 are assumed to be the same. A generalization of the IMTR strategy to cases where these dimensions are not the same and when the cost function 3 is nonquadratic can be made. See, for instance, our paperl that addresses optimal control of a transmission clutch on the basis of a high-order model. The treatment of convergence in such problems could be considerably more involved.

Remark 3. Note that $d$ is a function of time only and not of state; this leads to simple and fast updates, as the low-fidelity model remains linear with time-dependent disturbance input. If $d$ could be chosen as a function of state in Equation 2, a trivial choice $d=f\left(x_{h}, u\right)-A x_{l}-B u$ would provide a perfect match between the low-fidelity and high-fidelity models; however, this choice, clearly, does not address the underlying challenge of solving the optimal control problem. 


\section{3 | ITERATIVE MODEL \\ AND TRAJECTORY REFINEMENT CONVERGENCE}

In this section, we demonstrate that under appropriate assumptions, the iterations of the IMTR algorithm are expected to converge. While this analysis does not demonstrate that the iterations converge to the optimal solution for the high-fidelity model (and in general, examples where this is not true can be constructed), this convergence is clearly a desirable property because it implies that iterations do not diverge.

At the iteration $n$, the optimal control for the low-fidelity model is given by

$$
u^{n}=-R^{-1} B^{T} P x_{l}^{n}+R^{-1} B^{T} r^{n},
$$

while, with this optimal control, the closed-loop low-fidelity model is given by

$$
\dot{x}_{l}^{n}=\left(A-B R^{-1} B^{T} P\right) x_{l}^{n}+B R^{-1} B^{T} r^{n}+d^{n} .
$$

We define the $\lambda_{1}$-norm and $\lambda_{2}$-norm for a function $g(\cdot) \in$ $C^{0}([0, T])$ as follows:

$$
\begin{gathered}
\|g\|_{\lambda_{1}}=\sup _{t \in[0, T]} e^{-\lambda t}\|g(t)\|, \\
\|g\|_{\lambda_{2}}=\sup _{t \in[0, T]} e^{-\lambda(T-t)}\|g(t)\|,
\end{gathered}
$$

where $\lambda>0$. Note that the sup-norm overbounds and underbounds the $\lambda_{1}$-norm and $\lambda_{2}$-norm for a function $g(\cdot) \in$ $C^{0}([0, T])$ as follows:

$$
\begin{aligned}
& e^{-\lambda T}\|g\|_{\infty} \leqslant\|g\|_{\lambda_{1}} \leqslant\|g\|_{\infty}, \\
& e^{-\lambda T}\|g\|_{\infty} \leqslant\|g\|_{\lambda_{2}} \leqslant\|g\|_{\infty},
\end{aligned}
$$

where the sup-norm is defined as follows:

$$
\|g\|_{\infty}=\sup _{t \in[0, T]}\|g(t)\| .
$$

While, in principle, it is possible to develop results without introducing the norms 12 and 13, as they are equivalent to the standard sup-norm; the use of the norms 12 and 13 simplifies the application of the contraction mapping-type arguments in the convergence analysis and makes these arguments more elegant. An added advantage of using the norms 12 and 13 is that one can try to adjust the parameter $\lambda$ to satisfy the sufficient conditions for convergence.

The subsequent development of various bounds proceeds under the following assumption.

Assumption 1. Let $f: \mathbb{R}^{n} \times \mathbb{R}^{m} \rightarrow \mathbb{R}^{n}$ in Equation 1 be a globally Lipschitz continuous function so that

$$
\|f(x, u)-f(y, v)\| \leqslant L_{1}\|x-y\|+L_{2}\|u-v\|
$$

for all $x, y \in \mathbb{R}^{n}$ and $u, v \in \mathbb{R}^{m}$.
The derivation of conditions for IMTR convergence consists of several steps that are now explained at a high level. The reader is referred to Appendix A for details.

On the basis of Equations 1, 2, and 8, the bound on the change in the disturbance between two consecutive iterations is given by

$$
\begin{aligned}
\left\|d^{n+1}-d^{n}\right\|_{\lambda_{1}} \leqslant & m_{1}\left\|d^{n}-d^{n-1}\right\|_{\lambda_{1}}+m_{2}\left\|r^{n}-r^{n-1}\right\|_{\lambda_{2}} \\
& +m_{3}\left\|x_{l}^{n}-x_{l}^{n-1}\right\|_{\lambda_{1}}+m_{4}\left\|x_{h}^{n}-x_{h}^{n-1}\right\|_{\lambda_{1}},
\end{aligned}
$$

where $m_{1}>0, m_{2}>0, m_{3}>0$, and $m_{4}>0$ are appropriately defined. Then on the basis of Equation 7, it can be shown that there exists $m_{5}>0$ such that

$$
\left\|r^{n+1}-r^{n}\right\|_{\lambda_{2}} \leqslant m_{5}\left\|d^{n+1}-d^{n}\right\|_{\lambda_{1}} .
$$

In turn, on the basis of Equation 11, it can be shown that there exists $m_{6}>0$ and $m_{7}>0$ such that

$$
\left\|x_{l}^{n+1}-x_{l}^{n}\right\|_{\lambda_{1}} \leqslant m_{6}\left\|d^{n+1}-d^{n}\right\|_{\lambda_{1}}+m_{7}\left\|r^{n+1}-r^{n}\right\|_{\lambda_{2}} .
$$

It follows from Equations 1 and 5 that

$$
\left\|x_{h}^{n+1}-x_{h}^{n}\right\|_{\lambda_{1}} \leqslant m_{8}\left\|r^{n+1}-r^{n}\right\|_{\lambda_{2}}+m_{9}\left\|x_{l}^{n+1}-x_{l}^{n}\right\|_{\lambda_{1}} .
$$

From the inequalities 15 to 18 , it follows that there exists $\alpha>0$ such that

$$
\left\|d^{n+1}-d^{n}\right\|_{\lambda_{1}} \leqslant \alpha\left\|d^{n}-d^{n-1}\right\|_{\lambda_{1}} .
$$

The value of $\alpha$ is given by Equation A14 in Appendix A. The following result can now be stated.

Proposition 1. Suppose $\alpha \in(0,1)$, where $\alpha$ is given by Equation A14. Then the iterations of the IMTR algorithm converge. Specifically, $\lim _{n \rightarrow \infty} d^{n}=d^{\infty}, \lim _{n \rightarrow \infty} r^{n}=r^{\infty}, \lim _{n \rightarrow \infty} x_{l}^{n}=x_{l}^{\infty}$ and $\lim _{n \rightarrow \infty} x_{h}^{n}=x_{h}^{\infty}$ uniformly on $[0, T]$ for appropriate continuous functions, $d^{\infty}, r^{\infty}, x_{l}^{\infty}$ and $x_{h}^{\infty}$, respectively. Moreover, $\lim _{n \rightarrow \infty}\left\|x_{h}^{n}-x_{l}^{n}\right\|_{\infty}=0, J_{l}^{\infty}=\lim _{n \rightarrow \infty} J\left(x_{l}^{n}\right)=\lim _{n \rightarrow \infty} J\left(x_{h}^{n}\right)=J_{h}^{\infty}$.

Proof. See Appendix $B$.

Remark 4. The condition in Proposition 1 is sufficient and requires the computation of $\alpha$. We generally expect that small values of $k$ are conducive to being able to satisfy this condition and achieve IMTR convergence. For the example in Section 5.3, we provide a numerical study of the dependence of $\alpha$ on $k$, and we leave further study of this dependence to future work.

Remark 5. Proceeding a bit further, it is also possible to obtain a characterization of the low-fidelity model cost change over 1 iteration, $J_{l}^{n+1}-J_{l}^{n}$ as a quadratic function of $k>0$ in Equation 8. Specifically, the update equations can be written as follows:

$$
\begin{aligned}
& d^{n+1}(t)=d^{n}(t)+k \dot{e}^{n}(t), \\
& r^{n+1}(t)=r^{n}(t)+k \phi^{n}(t),
\end{aligned}
$$



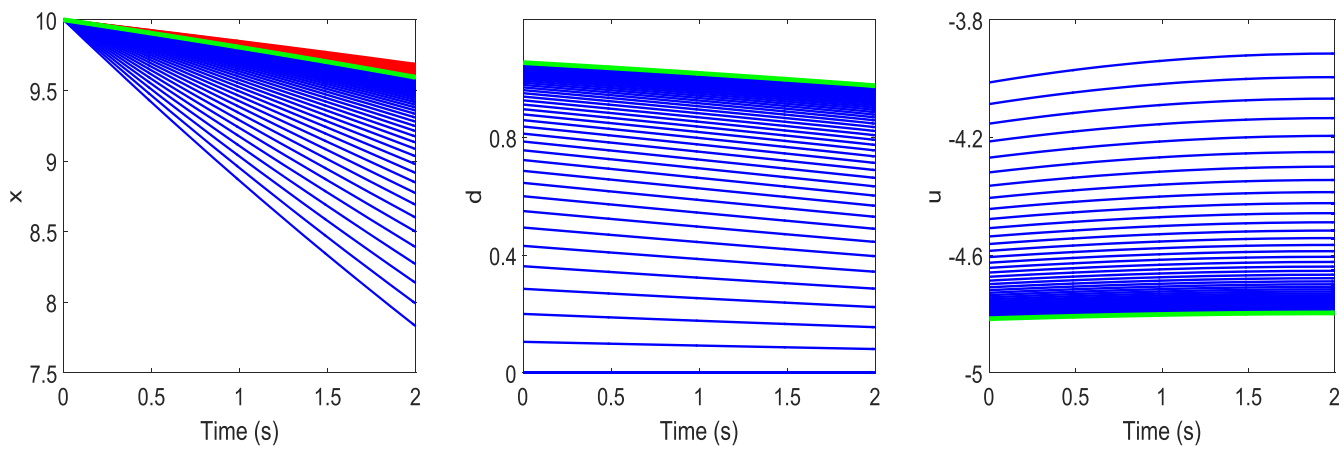

FIGURE 1 An example to demonstrate the sufficient condition in Proposition 1. Left: High and low-fidelity model trajectories. Middle: Disturbance trajectories. Right: Control trajectories [Colour figure can be viewed at wileyonlinelibrary.com]

with $\phi^{n}(t)$ satisfying the following differential equation,

$$
-\dot{\phi}^{n}=\tilde{A}^{T} \phi^{n}-\tilde{B} \dot{e}^{n}, \quad \phi^{n}(T)=0,
$$

where $\dot{e}^{n}=\dot{x}_{h}^{n}-\dot{x}_{l}^{n}$ and $\tilde{A}, \tilde{B}$ are defined in Appendix $A$. On the basis of Equations 4 to 7, it can be shown (see, eg, Boltyanski $^{16}$, p. 76)

$$
\begin{gathered}
J_{l}^{n+1}-J_{l}^{n}=-k^{2} \int_{0}^{T}\left[\phi^{n}(\tau)^{T} \dot{e}^{n}(\tau)+\frac{1}{2} \phi^{n}(\tau)^{T} B R^{-1} B^{T} \phi^{n}(\tau)\right] d \tau \\
-k\left[\phi^{n}(0)^{T} x_{l}^{n}(0)+\int_{0}^{T}\left[r^{n}(\tau)^{T} \dot{e}^{n}(\tau)+\phi^{n}(\tau)^{T} d^{n}(\tau)\right.\right. \\
\left.\left.+\phi^{n}(\tau)^{T} B R^{-1} B^{T} r^{n}(\tau)\right] d \tau\right] .
\end{gathered}
$$

The ability to easily predict the change in the low-fidelity model cost as a function of $k$ may be exploited in strategies for online selection of $k$. We leave the development of such strategies to future work, while our subsequent examples use a constant $k$.

Remark 6. The IMTR is a computational strategy and convergence may not be expected for all types of problems. Computational studies and further numerical analysis need to be undertaken to understand whether highly divergent, in particular, chaotic systems may be amenable to this strategy.

\section{1 | Example}

We now provide an example to demonstrate the sufficient condition in Proposition 1. In this example, the high-fidelity model is a scalar nonlinear system, while the corresponding low-fidelity model is the linearization of the high-fidelity model at the origin plus a disturbance. Specifically, the high-fidelity model and the corresponding low-fidelity model are given as

$$
\begin{gathered}
\dot{x}_{h}=-0.1 \sin x_{h}+0.05 u, \\
\dot{x}_{l}=-0.1 x_{l}+0.05 u+d .
\end{gathered}
$$

The cost function is given by Equation 3 with $Q=1, R=1$, and $K_{f}=10$. One set of parameters that satisfies the sufficient condition in Proposition 1 is given by

$$
\begin{aligned}
\lambda & =0.6, \\
k & =0.1, \\
T & =2,
\end{aligned}
$$

which yields

$$
\alpha=0.9635 \in(0,1) .
$$

The reader is referred to Appendix C for details. Thus, the sufficient condition in Proposition 1 holds and we also confirm the convergence by numerical simulations. Figure 1 shows the evolution of trajectories during the iterations of IMTR. The blue and red trajectories correspond to the lowand high-fidelity models, respectively, while the final trajectory after 100 iterations is shown in green.

\section{4 | NEAR-OPTIMAL SOLUTION B Y IMTR}

The control trajectory obtained by IMTR may be different from the optimal control for the high-fidelity model, obtained, eg, by the maximum principle and the solution of the resulting two-point boundary value problem. By the algorithm modification in Remark 1, a cost decrease for the high-fidelity model is guaranteed after each control update. In several examples, we have noticed that the solution of IMTR is close to the solution of the original optimal control problem. Motivated by this observation, we provide the following conditions supporting the expectation for the near-optimality of IMTR solution under certain assumptions.

Proposition 2. Suppose the nonlinear high-fidelity model is of the following form:

$$
\dot{x}_{h}=A x_{h}+B u+d_{h}\left(x_{h}, t\right),
$$

where $A$ and $B$ are the same as in Equation 2 and that there exists $\bar{d}(t)$ such that

$$
\left\|d_{h}\left(x_{h}, t\right)-\bar{d}(t)\right\| \leqslant \epsilon,
$$


for some $\epsilon>0$ and for all values of $x_{h}$. Then, assuming IMTR iterations converge in the sense of Proposition 1, the deviation of the optimal cost (Equation 3) from the cost (Equation 3) evaluated on the trajectories obtained by IMTR is $O(\epsilon)$ for $\epsilon$ sufficiently small.

Proof. See Appendix D.

Remark 7. It is clear from the proof of Proposition 2 that Equation 22 needs to only hold for the values of $x_{h}$ in the set which bounds the optimal trajectory of the high-fidelity model and the trajectory to which IMTR converges. While we leave further investigation of conditions under which near-optimality of IMTR solutions can be assured to future research, in the next sections, we investigate 2 case studies to numerically show that near-optimal solutions can be obtained through IMTR techniques.

\section{5 | ORBITAL TRANSFER}

The problem of optimal control of a low-thrust spacecraft orbital transfer maneuver is used to compare the NLQ and IMTR approaches. A target orbit is a circular Earth orbit with radius $R_{0}$ and constant orbital angular velocity $\dot{\theta}=\sqrt{\frac{\mu}{R_{0}^{3}}}$. A spacecraft in a different initial orbit must be controlled to match the orbital radius and velocity of the target orbit at a fixed final time.

The high-fidelity model is the classical 2-body model, with the equations of motion written in polar coordinates as $\mathrm{Wie}^{17}$ :

$$
\begin{gathered}
\ddot{r}-r \dot{\theta}^{2}=-\frac{\mu}{r^{2}}+a_{r}, \\
r \ddot{\theta}+2 \dot{r} \dot{\theta}=a_{\theta},
\end{gathered}
$$

where $r$ is the distance from the center of the Earth to the spacecraft, $\theta$ is the polar angle, and $a_{r}$ and $a_{\theta}$ are the thrust accelerations in the radial and tangential directions, respectively.

The system state is

$$
x=\left[\begin{array}{l}
x_{1} \\
x_{2} \\
x_{3} \\
x_{4}
\end{array}\right],
$$

where $x_{1}=r, x_{2}=\dot{r}, x_{3}=\theta, x_{4}=\dot{\theta}$. The control is defined by the acceleration components,

$$
u=\left[\begin{array}{l}
a_{r} \\
a_{\theta}
\end{array}\right] .
$$

The low-fidelity model uses the linearized 2-body orbital equations of motion, known as the Clohessy-Wiltshire equations $^{3}$ in the form given in $\mathrm{Wie}^{17}$ :

$$
\begin{gathered}
\Delta \ddot{r}=3 n^{2} \Delta r+2 n R_{0} \Delta \dot{\theta}+a_{r}, \\
R_{0} \Delta \ddot{\theta}=-2 n \Delta \dot{r}+a_{\theta} .
\end{gathered}
$$

This is a linear model with state variables $\Delta r$ and $\Delta \theta$ representing perturbations of the spacecraft radial distance and polar angle from an (imagined) spacecraft on the target circular orbit. The state trajectory of the (imagined) spacecraft on the target circular orbit is denoted by $\dot{x}_{\text {target }}$.

\section{1 | Maximum principle solution}

The optimal orbital transfer control problem is first solved using NLQ control. A finite horizon cost is defined

$$
\begin{aligned}
J= & \frac{1}{2}\left(x(T)-x_{T}\right)^{T} K_{f}\left(x(T)-x_{T}\right) \\
& +\frac{1}{2} \int_{0}^{T}\left[\left(x(\tau)-x_{T}\right)^{T} Q\left(x(\tau)-x_{T}\right)+u(\tau)^{T} R u(\tau)\right] d \tau,
\end{aligned}
$$

where $T$ is the final time and $x_{T}$ is the target state. To minimize the cost, the maximum principle is used. The Hamiltonian for the minimization problem is

$$
\begin{aligned}
H= & \frac{1}{2}\left(x-x_{T}\right)^{T} Q\left(x-x_{T}\right)+\frac{1}{2} u^{T} R u+p_{1} x_{2} \\
& +p_{2}\left(x_{1} x_{4}^{2}-\frac{\mu}{x_{1}^{2}}+a_{r}\right)+p_{3} x_{4}+p_{4} \frac{1}{x_{1}}\left(-2 x_{2} x_{4}+a_{\theta}\right),
\end{aligned}
$$

where $p_{1}, p_{2}, p_{3}$, and $p_{4}$ are the adjoint variables. The first-order necessary conditions for optimality are

$$
\left(\frac{\partial H}{\partial u}\right)^{T}=R u+\left[\begin{array}{l}
p_{2} \\
\frac{p_{4}}{x_{1}}
\end{array}\right]=0 .
$$

Therefore, the optimal control is given by

$$
u=-R^{-1}\left[\begin{array}{l}
p_{2} \\
\frac{p_{4}}{x_{1}}
\end{array}\right] .
$$

The adjoint equations are

$$
\begin{aligned}
\dot{p} & =-\left(\frac{\partial H}{\partial x}\right)^{T} \\
& =-Q\left(x-x_{T}\right)-\left[\begin{array}{l}
p_{2} x_{4}^{2}+2 \frac{p_{2} \mu}{x_{1}^{3}}-\frac{p_{4}}{x_{1}^{2}}\left(-2 x_{2} x_{4}+a_{\theta}\right) \\
p_{1}-2 \frac{p_{4}}{x_{1}} x_{4} \\
0 \\
2 p_{2} x_{1} x_{4}+p_{3}-2 p_{4} \frac{x_{2}}{x_{1}}
\end{array}\right] .
\end{aligned}
$$

The transversality conditions are

$$
p(T)=K_{f}\left(x(T)-x_{T}\right) .
$$

The two-point boundary value problem is solved for $x(0)$ and $p(0)$ such that Equation 30 holds. 


\section{2 | Iterative model and trajectory refinement solution}

The IMTR control solution to the orbital transfer problem is found using the approach described in Section 2. The high-fidelity model is given by Equations 23 and 24 with a state vector $x_{h}$ given by Equation 25. The low-fidelity model is given by Equations 27 and 28. The low-fidelity state is given by perturbations of the spacecraft from the target,

$$
x_{l}=\left[\begin{array}{c}
\Delta r \\
\Delta r \\
\Delta \theta \\
\Delta \dot{\theta}
\end{array}\right] .
$$

Consistently, with the low-fidelity model based on the Clohessy-Wiltshire equations 27 and 28, the disturbance to be added to the low-fidelity model is based on the difference in the dynamics of the perturbed state,

$$
d^{n+1}(t)=d^{n}(t)+k\left[\left(\hat{\dot{x}}_{h}^{n}(t)-\dot{x}_{\text {target }}(t)\right)-\hat{\hat{x}}_{l}^{n}(t)\right],
$$

where $x_{\text {target }}(t)$ represents the state trajectory of an (imagined) spacecraft on the target circular orbit.

The low- and high-fidelity model costs are calculated using Equation 29 with $x$ given by $\left(x_{h}-x_{\text {target }}\right)$ and $x_{l}$, respectively. The IMTR algorithm is applied with the modification described in Remark 1 of Section 2, ie, only the disturbance - not the control-is updated during iterations in which the control update would increase the high-fidelity model cost.

\section{3 | Orbital transfer example}

Figures 2 to 6 show an example of the maximum principle and IMTR applied to the targeting problem with
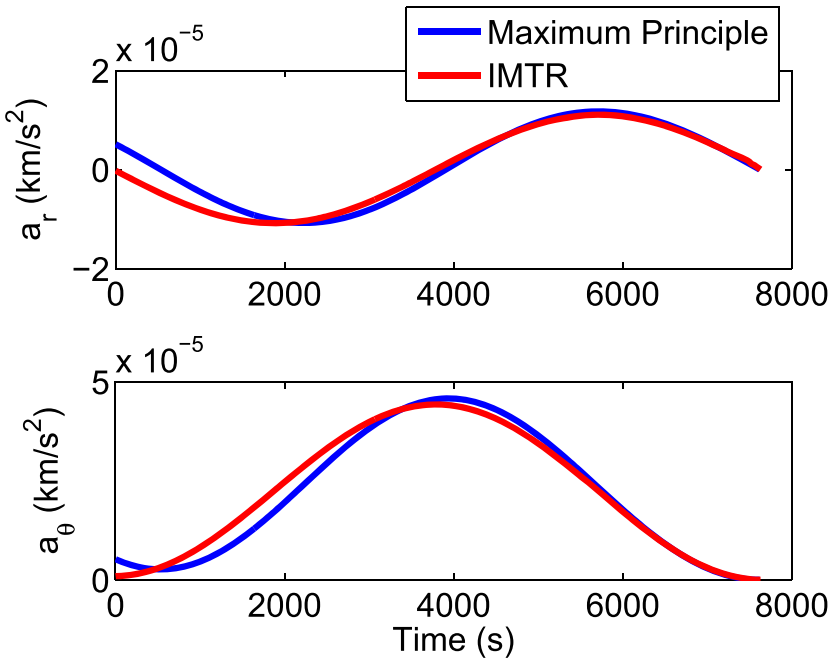

FIGURE 2 Commanded acceleration in orbital transfer problem [Colour figure can be viewed at wileyonlinelibrary.com]

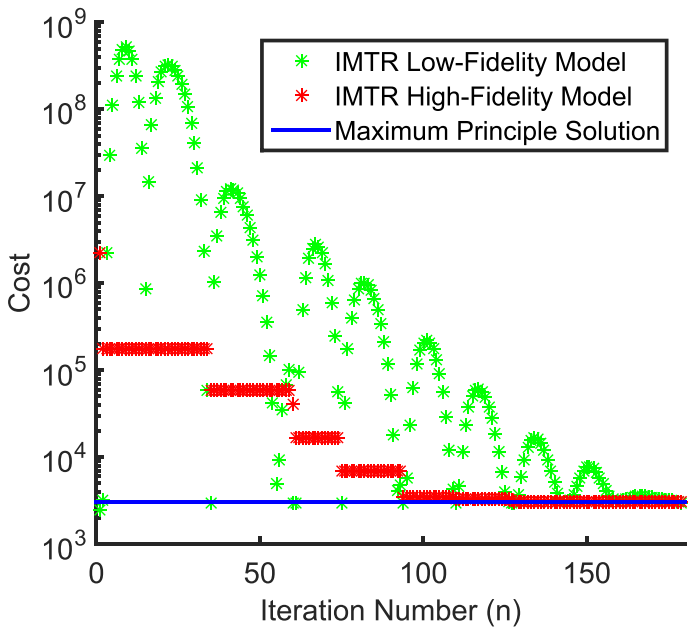

FIGURE 3 Cost in orbital transfer problem [Colour figure can be viewed at wileyonlinelibrary.com]

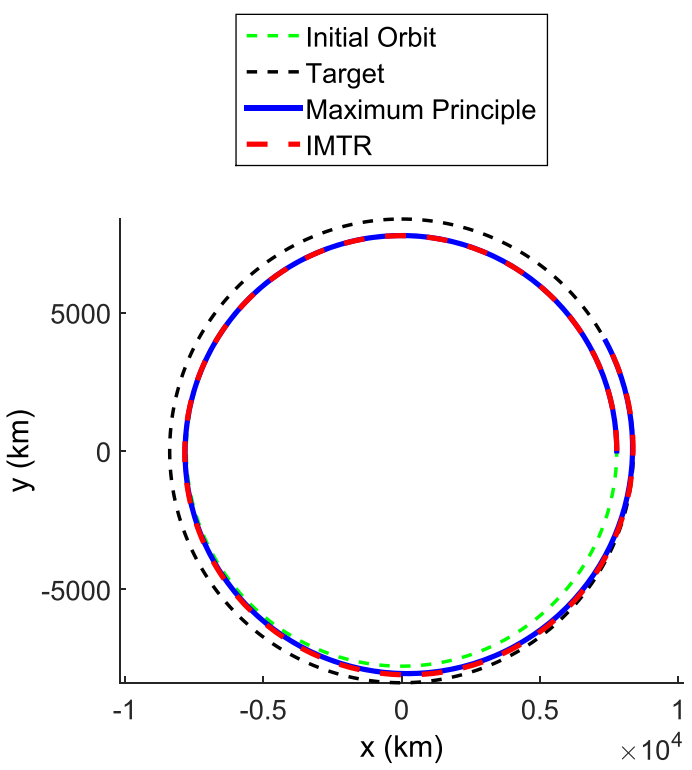

FIGURE 4 Planar $(x, y)$ trajectories in orbital transfer problem [Colour figure can be viewed at wileyonlinelibrary.com]

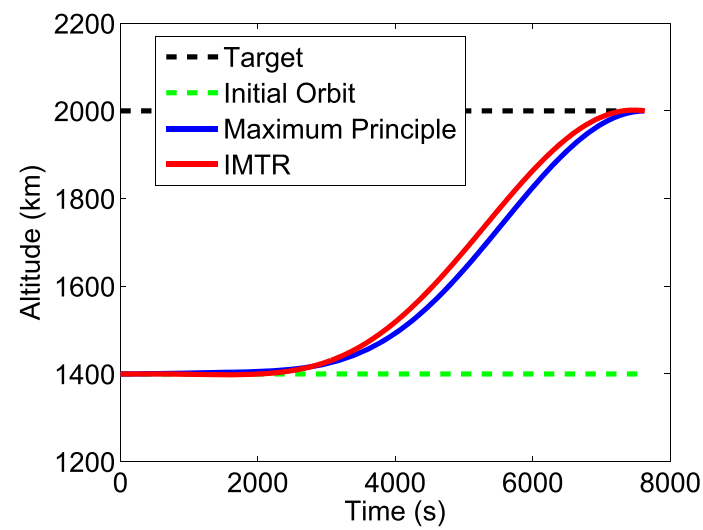

FIGURE 5 Altitude in orbital transfer problem [Colour figure can be viewed at wileyonlinelibrary.com] 


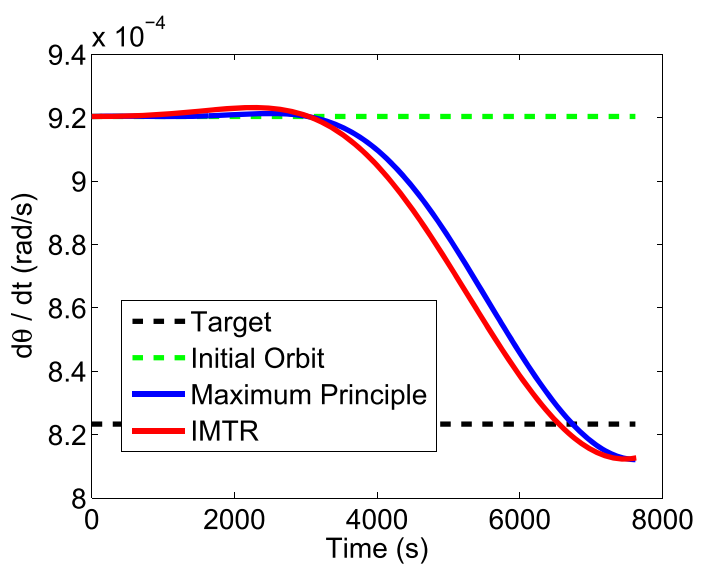

FIGURE 6 Angular rate in orbital transfer problem [Colour figure can be viewed at wileyonlinelibrary.com]

$$
\begin{gathered}
x_{0}=\left[\begin{array}{c}
R_{e}+1400 \\
0 \\
0 \\
\sqrt{\frac{\mu}{\left(R_{e}+1400\right)^{3}}}
\end{array}\right], \\
x_{T}=\left[\begin{array}{c}
R_{e}+2000 \\
0 \\
0 \\
\sqrt{\frac{\mu}{\left(R_{e}+2000\right)^{3}}}
\end{array}\right],
\end{gathered}
$$

ie, a spacecraft transferring from a $1400 \mathrm{~km}$ circular orbit to a $2000 \mathrm{~km}$ circular orbit. Note that a $600 \mathrm{~km}$ orbit transfer maneuver is large and the Clohessy-Wiltshire equations alone are not accurate at such distances to plan an accurate maneuver. As we will demonstrate, accurate maneuvers can be achieved using IMTR.

The transfer time $T$ is the orbit period of the target, $T=$ 7,631 seconds $=2.12$ hours. The penalty on the final state is

$$
K_{f}=\left[\begin{array}{llll}
1 & 0 & 0 & 0 \\
0 & 1 & 0 & 0 \\
0 & 0 & 0 & 0 \\
0 & 0 & 0 & 1
\end{array}\right],
$$

which avoids penalizing the polar angle $x_{3}=\theta$. This represents an orbit-raising problem, with no constraint on the final angular position of the spacecraft. In this example, $Q$ is set to 0 , because nonzero $Q$ makes the adjoint equations stiff and $R$ is

$$
R=\left[\begin{array}{cc}
10^{9} & 0 \\
0 & 10^{9}
\end{array}\right]
$$

which was found to yield solutions with low thrust accelerations (existing electric propulsion systems typically produce less than $1 \mathrm{~N}$ of thrust, ${ }^{18,19}$ while developmental systems may be able to produce up to $15 \mathrm{~N}^{20}$ ). The parameter value $k=0.2$ was selected to limit the change in $d$ from one iteration to the next.
Figure 2 shows the optimal control inputs $a_{r}$ and $a_{\theta}$ calculated by both the maximum principle and the IMTR strategy. The results are similar, but not identical.

Figure 3 shows the cost (Equation 29) calculated by the low- and high-fidelity models during each iteration of the IMTR algorithm, compared to the optimal cost calculated by the maximum principle. The low-fidelity model cost varies as the disturbance updates improve the low-fidelity model match over several iterations. The high-fidelity model cost decreases in several steps.

In this example, the IMTR algorithm termination criterion was that $J_{h}$ change by less than $1 \%$ of its final value over the previous 2 decreasing steps. This criterion was satisfied after 164 iterations. This number of iterations is reasonable compared to other iterative trajectory optimization methods. For example, Betts ${ }^{21}$ used an SQP algorithm to solve a low-thrust orbital trajectory problem with 2 formulations requiring 125 and 263340 iterations, respectively.

The control solution from the maximum principle approach had a maximum total acceleration of $0.0458 \mathrm{~m} / \mathrm{s}^{2}$, where total acceleration is

$$
a_{\text {total }}(t)=\sqrt{a_{r}(t)^{2}+a_{\theta}(t)^{2}} .
$$

This maximum acceleration corresponds to a thrust of $13.7 \mathrm{~N}$ for a $300 \mathrm{~kg}$ spacecraft. The control solution from the IMTR approach had a maximum total acceleration of $0.0443 \mathrm{~m} / \mathrm{s}^{2}$. This maximum acceleration corresponds to a thrust of $13.3 \mathrm{~N}$ for a $300 \mathrm{~kg}$ spacecraft. Both methods achieved approximately the same optimal cost; $J=3,033$ for the maximum principle solution and $J=3,072$ for the IMTR solution.

Figure 4 shows the orbital trajectories resulting from the maximum principle and the IMTR solution. The 2 solutions overlap closely.

Figures 5 and 6 show the resultant time-histories of orbit altitude and angular rate from the maximum principle and IMTR. The results are very similar for both methods; the maximum principle yields a slightly slower solution, but the final states are very similar. Both methods reach the target radius but miss the target angular rate by about $1 \cdot 10^{-5} \mathrm{rad} / \mathrm{s}$. This is the result of our choice of $Q, R$, and $K_{f}$; a small miss in $\dot{\theta}$ achieved the lowest total cost. A more accurate solution, at the expense of a higher control cost, could be obtained by adjusting these matrices.

Figures 7 and 8 show the results of the maximum principle and the IMTR strategy for a range of initial conditions. The orbital transfer problem with a target altitude of $2000 \mathrm{~km}$ was solved for initial altitudes ranging from 1000 to $1900 \mathrm{~km}$. In the IMTR solutions, the parameter value $k=0.5$ was used. All other problem parameters were maintained at the values given above. Each problem was terminated when $J_{h}$ changed by less than $1 \%$ over 2 sequential steps. As expected, the cost and the maximum acceleration decreases for initial conditions 


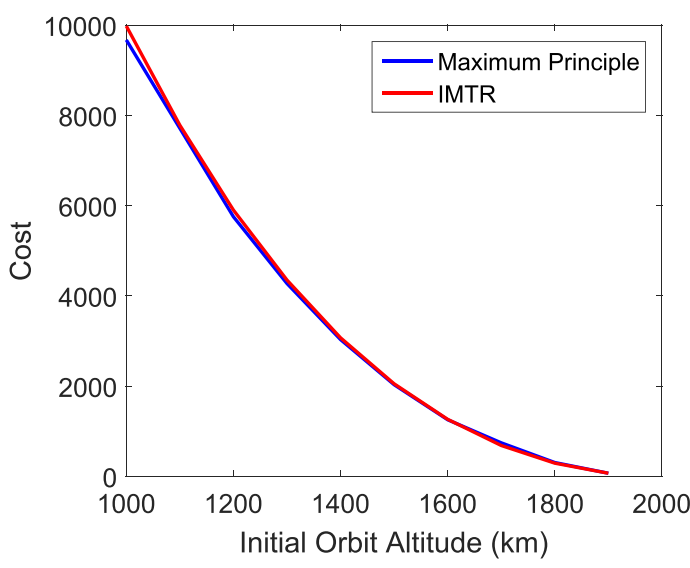

FIGURE 7 Cost vs initial altitude in orbital transfer problem [Colour figure can be viewed at wileyonlinelibrary.com]

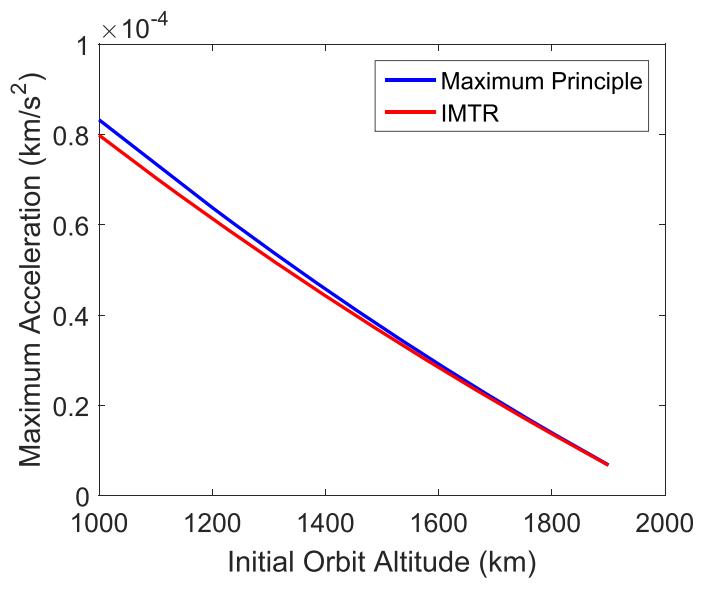

FIGURE 8 Maximum acceleration vs initial altitude in orbital transfer problem [Colour figure can be viewed at wileyonlinelibrary.com]

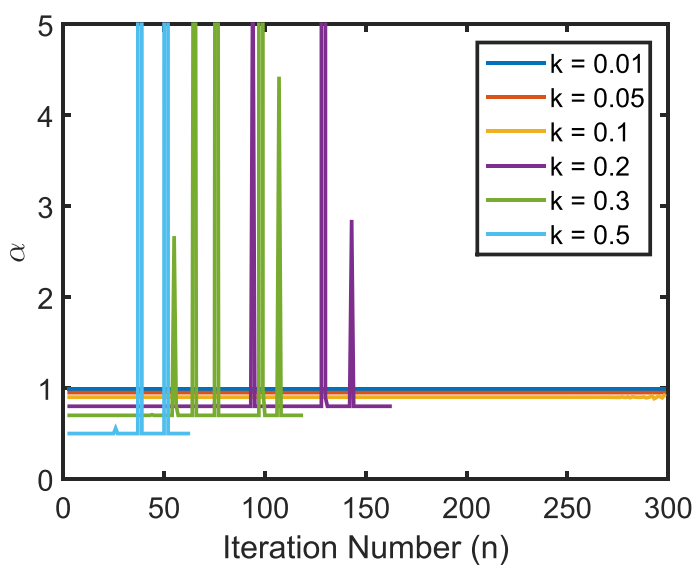

FIGURE $9 \alpha$ vs iteration number in orbital transfer problem [Colour figure can be viewed at wileyonlinelibrary.com]

closer to the target orbit. The maximum principle and IMTR solutions are very similar; the maximum principle results in slightly lower cost and slightly higher maximum acceleration for the cases with the lowest initial altitude.
As per Proposition 1, the value of $\alpha$ in Equation 19 determines convergence of the IMTR algorithm. In the orbital transfer example in Figures 2 to 6, $\alpha$ varies as a function of iteration number $n$ and parameter $k$. Figure 9 shows the value of $\alpha$, calculated numerically using Equations 12 and A13 with $\lambda=2$ for this example. For $k \leqslant 0.1$, we find $\alpha \in(0,1)$ on every iteration. For $k=0.2,0.3,0.5$, the value of $\alpha$ is generally in this range, but there are exceptions on a few isolated iterations. In these cases, the process returns to $\alpha<1$ on the next iteration; these exceptions do not prevent convergence. The IMTR algorithm converged for all values of $k$ shown in Figure 9, with a faster rate of convergence for larger values of $k$.

\section{6 | EARTH-MOON $L_{4}$ TARGETING}

As a second example, we apply the IMTR strategy to the problem of optimally controlling a spacecraft near the $L_{4}$ Lagrange point of the Earth-Moon system. ${ }^{17}$ The 5 Lagrange points are the locations in a 3-body orbital system where a small body can maintain a constant position with respect to 2 larger bodies, as gravitational and centripetal forces cancel. A spacecraft can orbit a Lagrange point and remain in constant alignment with the primary bodies, which makes the Lagrange points useful locations for many space missions.

The nonlinear equations of the planar circular restricted 3 -body model ${ }^{17}$ are the high-fidelity model of the system,

$$
\begin{aligned}
& \ddot{x}-2 \omega_{0} \dot{y}-\omega_{0}^{2} x=\frac{\mu_{1}\left(x-D_{1}\right)}{r_{1}^{3}}-\frac{-\mu_{2}\left(x+D_{2}\right)}{r_{2}^{3}}+\eta_{x}+a_{x}, \\
& \ddot{y}+2 \omega_{0} \dot{x}-\omega_{0}^{2} y=\frac{\mu_{1} y}{r_{1}^{3}}-\frac{-\mu_{2} y}{r_{2}^{3}}+\eta_{y}+a_{y},
\end{aligned}
$$

where the coordinate frame is rotating with constant velocity $\omega_{0}$ about the center of mass of the Earth-Moon system, $D_{1}$ is the distance to the Earth from the center along the positive $x$ axis, $D_{2}$ is the distance to the Moon from the center along the negative $x$ axis, and $\mu_{1}$ and $\mu_{2}$ are the constant gravity parameters of the Earth and Moon, respectively. The perturbations $\eta_{x}$ and $\eta_{y}$ are added to represent unknown system behavior, such as control disturbances, solar radiation pressure perturbations, and gravitational perturbations. The accelerations $a_{x}$ and $a_{y}$ are the control inputs.

The linearized restricted 3-body orbital dynamics for perturbations from $L_{4}$ equilibrium ${ }^{17}$ are the low-fidelity model of the system,

$$
\begin{aligned}
& \Delta \ddot{x}=2 \omega_{0} \Delta \dot{y}+\frac{3}{4} \omega_{0}^{2} \Delta x+\frac{3 \sqrt{3}}{2}\left(\rho-\frac{1}{2}\right) \omega_{0}^{2} \Delta y+a_{x}, \\
& \Delta \ddot{y}=-2 \omega_{0} \Delta \dot{x}+\frac{3 \sqrt{3}}{2}\left(\rho-\frac{1}{2}\right) \omega_{0}^{2} \Delta x+\frac{9}{4} \omega_{0}^{2} \Delta y+a_{y},
\end{aligned}
$$

where $\Delta x$ and $\Delta y$ are the components of the position vector of the spacecraft relative to the Lagrange point and $\rho$ is the mass ratio of the Earth-Moon system, 


$$
\rho=\frac{M_{\text {Moon }}}{M_{\text {Earth }}+M_{\text {Moon }}} .
$$

Comparing the 2 models, the high-fidelity model alone is more difficult and computationally expensive to optimize because of its nonlinear structure. The low-fidelity model is amenable to LQ optimal control, but the solution becomes less accurate as the state moves away from $L_{4}$. In the simulations below, the IMTR strategy converges on a solution to the high-fidelity problem from initial points more than $14000 \mathrm{~km}$ away from $L_{4}$.

The following examples show an optimal control problem in which the objective is to control the spacecraft from a perturbed initial state to the $L_{4}$ point. The initial state perturbation from $L_{4}$ is

$$
x_{l}(0)=\left[\begin{array}{c}
\Delta x \\
\Delta y \\
\Delta \dot{x} \\
\Delta \dot{y}
\end{array}\right] .
$$

A cost function of form 29 is defined using the high-fidelity state,

$$
x_{h}=\left[\begin{array}{c}
x \\
y \\
\dot{x} \\
\dot{y}
\end{array}\right] .
$$

The final time is fixed at 24 hours. The acceleration perturbations are $\eta_{x}=1 \cdot 10^{-9} \mathrm{~km} / \mathrm{s}^{2}$ and $\eta_{y}=2 \cdot 10^{-9} \mathrm{~km} / \mathrm{s}^{2}$. The cost to be minimized is given by Equation 3 with $K_{f}=I_{4}, Q=I_{4}$, and

$$
R=\left[\begin{array}{cc}
10^{14} & 0 \\
0 & 10^{14}
\end{array}\right],
$$

which was found to yield low thrust acceleration solutions. The parameter value $k=0.1$ was selected.

Figures 10 to 12 show the IMTR strategy applied to the targeting problem with initial conditions $x(0)=5,000 \mathrm{~km}$, $y(0)=5,000 \mathrm{~km}$, in the rotating frame, relative to $L_{4}$. The IMTR algorithm was terminated when the cost (Equation 3) varied by less than $10 \%$ over 3 iterations, and the final cost

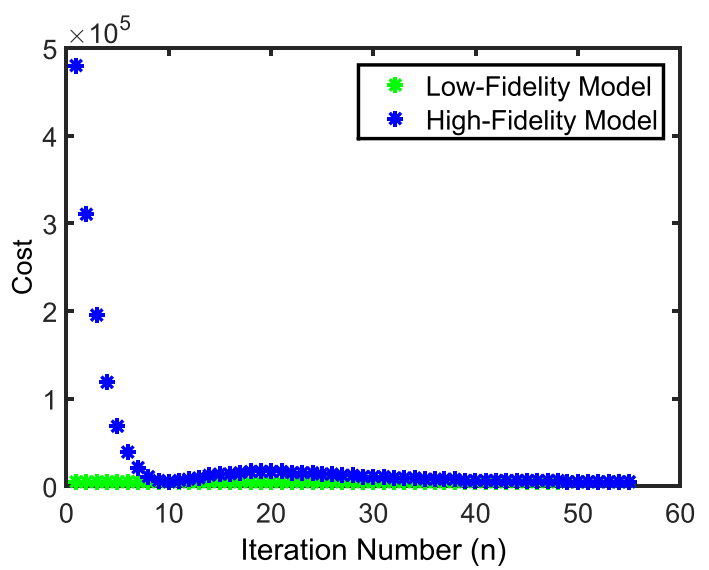

FIGURE 10 Cost in Earth-Moon $L_{4}$ targeting problem [Colour figure can be viewed at wileyonlinelibrary.com]

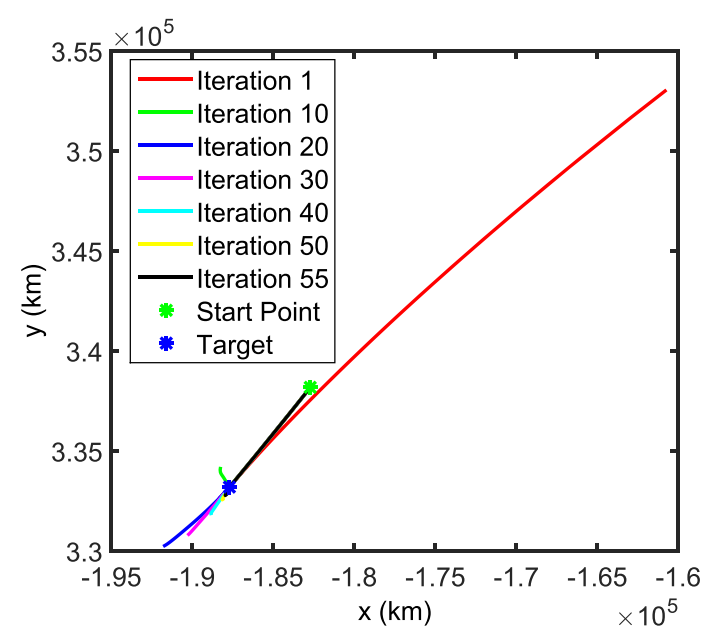

FIGURE 11 Trajectories in the rotating frame calculated during selected iterations of the IMTR algorithm in Earth-Moon $L_{4}$ targeting problem [Colour figure can be viewed at wileyonlinelibrary.com]

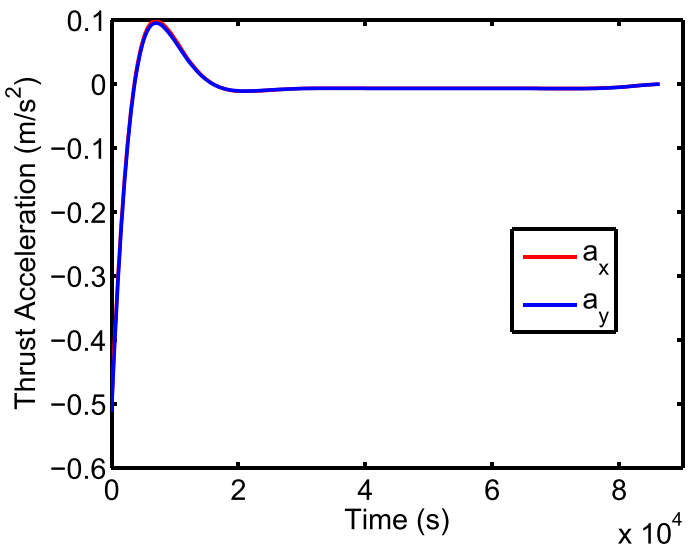

FIGURE 12 Thrust acceleration in Earth-Moon $L_{4}$ targeting problem [Colour figure can be viewed at wileyonlinelibrary.com]

was less than any previously calculated cost. This occurred after 55 iterations.

Figure 10 shows the costs calculated by the low- and high-fidelity models on each iteration of the IMTR algorithm. The cost decreases sharply over the first 10 iterations and then approaches the minimum more slowly over the next 45 iterations.

Figure 11 shows the calculated trajectories at various iterations of the IMTR algorithm. The $(x, y)$ reference frame rotates with the Earth-Moon system. The first trajectory initially approaches the target, but ends far from the target point, which indicates that the low-fidelity model does not solve the problem with this initial condition. However, accuracy improves over the iterations and the final trajectory approaches the target and does not diverge.

Figure 12 shows the solution to the problem, the commanded thrust acceleration. The accelerations in the $x$ and $y$ directions are nearly identical. 


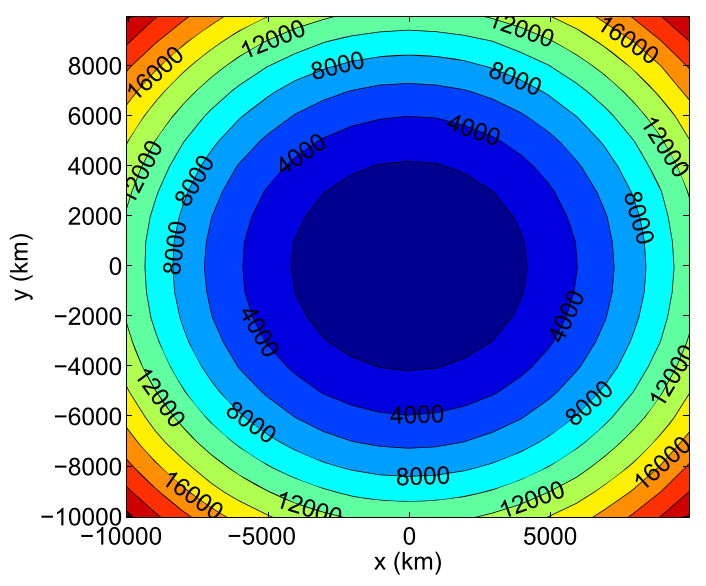

FIGURE 13 Calculated cost vs initial position relative to $L_{4}$ in Earth-Moon $L_{4}$ targeting problem [Colour figure can be viewed at wileyonlinelibrary.com]

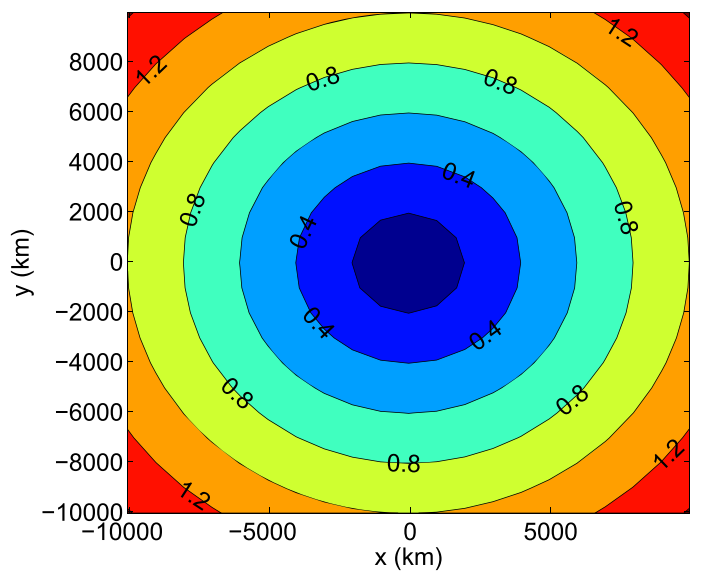

FIGURE 14 Maximum acceleration $\left(\mathrm{m} / \mathrm{s}^{2}\right)$ vs initial position relative to $L_{4}$ in Earth-Moon $L_{4}$ targeting problem [Colour figure can be viewed at wileyonlinelibrary.com]

Figures 13 to 14 show the results of the same approach for a range of initial conditions. The values of $\Delta x(0)$ and $\Delta y(0)$ varied from $-10050 \mathrm{~km}$ to $10050 \mathrm{~km}$ while $\Delta \dot{x}(0)$ and $\Delta \dot{y}(0)$ were held at 0 . The final time and the constants $\eta_{x}, \eta_{y}, K_{f}, Q$, and $R$ have the values given above. The IMTR method was used to calculate the optimal trajectory to the $L_{4}$ point. The algorithm converged for each case, with initial distances ranging from $50 \mathrm{~km}$ to $14213 \mathrm{~km}$ from $L_{4}$.

Figure 13 shows the minimum cost $J$ calculated by the IMTR approach for each initial position relative to the $L_{4}$ point. As expected, the cost increases with distance from $L_{4}$.

Figure 14 shows the maximum total acceleration required for the trajectory from each initial position, where total acceleration is

$$
a_{\text {total }}(t)=\sqrt{a_{x}(t)^{2}+a_{y}(t)^{2}} .
$$

The maximum acceleration ranges from $1.4 \mathrm{~m} / \mathrm{s}^{2}$ at the farthest initial conditions to $0.0105 \mathrm{~m} / \mathrm{s}^{2}$ at the nearest initial conditions. For a $300 \mathrm{~kg}$ spacecraft, these maximum accelerations correspond to maximum thrusts of $420 \mathrm{~N}$ and $3.15 \mathrm{~N}$, respectively.

\section{7 | CONCLUSIONS}

This paper presented an IMTR approach to spacecraft trajectory optimization using iterations of low- and high-fidelity models. Sufficient conditions for IMTR convergence were established. An example of low-thrust spacecraft orbital transfer was described and the IMTR solution was compared to NLQ control on the basis of the maximum principle. A second example of spacecraft control in the 3-body Earth-Moon system near the $L_{4}$ Lagrange point was also described and solved using the IMTR strategy.

Both examples exercised IMTR for a range of initial conditions, and in all cases, we have been able to obtain reasonable solutions. In the first example, IMTR calculated a solution very similar to the optimal solution on the basis of the maximum principle. In the second example, IMTR found control solutions for initial conditions far from the point about which the low-fidelity equations were linearized. These examples indicate that IMTR can be a useful strategy for many types of trajectory optimization problems with complex, nonlinear dynamics and low-fidelity model representations. In particular, IMTR can be used to warm start Newton-type two-point boundary value solvers that are based on necessary conditions for optimality; such solvers require a good initial guess to achieve convergence.

While our examples illustrate the potential for IMTR application to practical trajectory optimization problems, much room remains for further research on conditions under which convergence of IMTR takes place.

\section{ACKNOWLEDGEMENTS}

Ilya Kolmanovsky and Nan Li acknowledge support of the National Science Foundation Award CNS 1544844.

\section{REFERENCES}

1. Hudson J, Kolmanovsky IV, Hong J, et al. Iterative model and trajectory refinement for launch optimization of automotive powertrains. Proceedings of 15th IFAC Workshop on Control Applications of Optimization. Rimini, Italy; September 2012:147-151.

2. Gupta R, Hudson J, Bloch AM, Kolmanovsky IV. Optimal control of manifold filling during VDE mode transitions. Proceedings of the 52nd IEEE Conference on Decision and Control. Florence, Italy; 2013:2227-2232.

3. Clohessy WH, Wiltshire RS. Terminal guidance system for satellite rendezvous. J Aerosp Sci. 1960;27(9):653-658.

4. Hudson J, Kolmanovsky I. Iterative model refinement for orbital trajectory optimization. Proceedings of 2012 AAS/AIAA Space Flight Mechanics Meeting. Charleston, SC; 2012:1933-1950.

5. Hudson J, Kolmanovsky IV. Iterative model and trajectory refinement for attitude and shape control of a dumbbell spacecraft. 
Proceedings of 2013 AAS/AIAA Astrodynamics Specialist Conference. Hilton Head, South Carolina; Summer August 11, 2013. Paper AAS 13-890:2787-2796.

6. Frihauf P, Krstic M, Basar T. Finite-horizon LQ control for unknown discrete-time linear systems via extremum seeking. Proceedings of 51st IEEE Conference on Decision and Control (CDC), vol. 10-13. Maui, HI; December 2012:5717-5722. doi:10.1109/CDC.2012.6426052

7. Gunnarsson S, Norrlof M. On the design of ILC algorithms using optimization. Automatica. 2001;37(12):2011-2016. ISSN 0005-1098, 10.1016/S0005-1098(01)00154-6

8. Lee JH, Lee KS, Kim WC. Model-based iterative learning control with a quadratic criterion for time-varying linear systems. Autom. 2000;36(5):641-657. ISSN 0005-1098, 10.1016/S0005-1098(99)00194-6

9. Luus R. On the application of iterative dynamic programming to singular optimal control problems. IEEE Trans Autom Control. 1992;37(11):1802-1806. doi: 10.1109/9.173155

10. Hofer EP, Tibken B. An iterative method for the finite-time bilinear-quadratic control problem. J Optim Theory Appl. 1998;57(3):411-427. doi:10.1007/BF02346161

11. Ying YQ, Rao M, Sun Y. Suboptimal control for bilinear systems. Optimal Control Appl Methods. 1993;14(3):195-201. 10.1002/oca.4660140305

12. Aganovic $\mathrm{Z}$, Gajic $\mathrm{Z}$. The successive approximation procedure for finite-time optimal control of bilinear systems. IEEE Trans Autom Control. 1994;39(9):1932-1935. doi: 10.1109/9.317128

13. Lee S, Lee K. Bilinear systems controller design with approximation techniques. J Chungcheong Math Soc. 2005;18(1):101-116.

14. Conway BA. Spacecraft Trajectory Optimization. Cambridge: Cambridge University Press; 2010.

15. Isidori $\mathrm{A}$, Astolfi $\mathrm{A}$. Disturbance attenuation and $\mathrm{H} \infty$ control via measurement feedback in nonlinear systems. IEEE Trans Autom Control. 1992;37(9):1283-1293.

16. Boltyanski VG, Poznyak AS. The Robust Maximum Principle: Theory and Applications. New York: Birkhäuser Mathematics; 2012.

17. Wie B. Space Vehicle Dynamics and Control. Reston, VA: AIAA; 1998.

18. Polk JE, Brinza D, Kakuda RY, et al. Demonstration of the NSTAR ion propulsion system on the Deep Space One mission. Proceedings of the 27th International Electric Propulsion Conference. Pasadena, CA; October 2001. also Paper 01-075.

19. Patterson MJ, Benson SW. NEXT ion propulsion system development status and performance. Proceedings of the 43rd Joint Propulsion Conference and Exhibit. Cincinnati, OH; 2007. also Paper 2007-5199.

20. Florenz RE, Hall SJ, Gallimore AD, et al. First firing of a 100-kW nested-channel hall thruster. Proceedings of the 33rd International Electric Propulsion Conference. Washington, DC; 2013:27-40. IEPC-2013-394.

21. Betts JT. Very low-thrust trajectory optimization using a direct SQP method. J Comput Appl Math. 27-40;120.

How to cite this article: Hudson J, Gupta R, $\mathrm{Li} \mathrm{N}$, Kolmanovsky I. Iterative model and trajectory refinement for orbital trajectory optimization. Optim Control Appl Meth. 2017;38:1132-1147. https://doi.org/10.1002/oca.2319

\section{APPENDIX A: DERIVATION OF EQUATION 19}

We begin with the following facts.

1. For all $t \in[0, T]$, the following inequalities hold

$$
\begin{aligned}
\int_{0}^{t}\|g(\tau)\| d \tau & =\int_{0}^{t} e^{-\lambda \tau} e^{\lambda \tau}\|g(\tau)\| d \tau \\
& \leqslant\|g\|_{\lambda_{1}} \int_{0}^{t} e^{\lambda \tau} d \tau, \\
\int_{t}^{T}\|g(\tau)\| d \tau & =\int_{t}^{T} e^{-\lambda(T-\tau)} e^{\lambda(T-\tau)}\|g(\tau)\| d \tau \\
& \leqslant\|g\|_{\lambda_{2}} \int_{t}^{T} e^{\lambda(T-\tau)} d \tau .
\end{aligned}
$$

2. For all $t \in[0, T]$, the following inequalities hold

$$
\begin{array}{r}
e^{-\lambda t} \int_{0}^{t} e^{\lambda \tau} d \tau \leqslant \frac{1}{\lambda}, \\
e^{-\lambda(T-t)} \int_{t}^{T} e^{\lambda(T-\tau)} d \tau \leqslant \frac{1}{\lambda} .
\end{array}
$$

Define the following quantities,

$$
\begin{gathered}
c_{1}=\sup _{t \in[0, T]}\|\tilde{\mathrm{A}}(t)\|, \\
c_{2}=\sup _{t \in[0, T]}\left\|\tilde{\mathrm{A}}^{T}(t)\right\|, \\
c_{3}=\sup _{t \in[0, T]}\|\tilde{B}(t)\|, \\
c_{4}=\sup _{t \in[0, T]}\left\|K_{1}(t)\right\|, \\
c_{5}=\left\|K_{2}\right\|, \\
c_{6}=\left\|B K_{2}\right\|, \\
c_{7}=\sup _{t \in[0, T]} e^{-\lambda(T-t)} \int_{t}^{T} e^{\lambda \tau} d \tau, \\
c_{8}=\sup _{t \in[0, T]} e^{-\lambda t} \int_{0}^{t} e^{\lambda(T-\tau)} d \tau,
\end{gathered}
$$

where $\tilde{\mathrm{A}}=\left(A-B K_{1}\right), \tilde{B}=P, K_{1}=R^{-1} B^{T} P$ and $K_{2}=$ $R^{-1} B^{T}$. On the basis of Equations 1, 2, and 8, it follows that

$$
\begin{aligned}
d^{n+1}(t)-d^{n}(t)= & (1-k)\left(d^{n}(t)-d^{n-1}(t)\right) \\
& +k\left(f\left(x_{h}^{n}(t), u^{n}(t)\right)-f\left(x_{h}^{n-1}(t), u^{n-1}(t)\right)\right) \\
& +k\left(\left(A-B K_{1}(t)\right)\left(x_{l}^{n-1}(t)-x_{l}^{n}(t)\right)\right. \\
& \left.+B K_{2}\left(r^{n-1}(t)-r^{n}(t)\right)\right) .
\end{aligned}
$$

Multiplying both sides of this expression by $e^{-\lambda t}$ and taking the supremum over $[0, T]$ on both sides yields 


$$
\begin{aligned}
& \left\|d^{n+1}-d^{n}\right\|_{\lambda_{1}} \leqslant \underbrace{|1-k|}_{m_{1}}\left\|d^{n}-d^{n-1}\right\|_{\lambda_{1}}+\underbrace{|k| e^{\lambda T}\left(c_{6}+c_{5} L_{2}\right)}_{m_{2}}\left\|r^{n}-r^{n-1}\right\|_{\lambda_{2}} \\
& +\underbrace{|k|\left(c_{1}+c_{4} L_{2}\right)}_{m_{3}}\left\|x_{l}^{n}-x_{l}^{n-1}\right\|_{\lambda_{1}}+\underbrace{|k| L_{1}}_{m_{4}}\left\|x_{h}^{n}-x_{h}^{n-1}\right\|_{\lambda_{1}} .
\end{aligned}
$$

The norm of $r^{n+1}-r^{n}$ is bounded as follows on the basis of Equation 7,

$$
\begin{aligned}
r^{n+1}(t)-r^{n}(t)= & \int_{t}^{T} \tilde{\mathrm{A}}^{T}\left[r^{n+1}(\tau)-r^{n}(\tau)\right] d \tau \\
& +\int_{t}^{T} \tilde{B}\left[d^{n}(\tau)-d^{n+1}(\tau)\right] d \tau, \\
\left\|r^{n+1}(t)-r^{n}(t)\right\| \leqslant & c_{2} \int_{t}^{T}\left\|r^{n+1}(\tau)-r^{n}(\tau)\right\| d \tau \\
& +c_{3} \int_{t}^{T}\left\|d^{n+1}(\tau)-d^{n}(\tau)\right\| d \tau \\
\leqslant & c_{2}\left\|r^{n+1}-r^{n}\right\|_{\lambda_{2}} \int_{t}^{T} e^{\lambda(T-\tau)} d \tau \\
& +c_{3}\left\|d^{n+1}-d^{n}\right\|_{\lambda_{1}} \int_{t}^{T} e^{\lambda \tau} d \tau .
\end{aligned}
$$

Multiplying by $e^{-\lambda(T-t)}$ and taking the supremum over $[0, T]$ on both sides yields

$$
\left\|r^{n+1}-r^{n}\right\|_{\lambda_{2}} \leqslant \underbrace{\frac{c_{3} c_{7}}{1-\lambda^{-1} c_{2}}}_{m_{5}}\left\|d^{n+1}-d^{n}\right\|_{\lambda_{1}} .
$$

The difference $x_{l}^{n+1}-x_{l}^{n}$ is bounded as follows on the basis of Equation 11

$$
\begin{aligned}
x_{l}^{n+1}(t)-x_{l}^{n}(t)= & \int_{0}^{t} \tilde{\mathrm{A}}(\tau)\left[x_{l}^{n+1}(\tau)-x_{l}^{n}(\tau)\right] d \tau \\
& +\int_{0}^{t} B K_{2}\left[r^{n+1}(\tau)-r^{n}(\tau)\right] d \tau \\
& +\int_{0}^{t}\left[d^{n+1}(\tau)-d^{n}(\tau)\right] d \tau, \\
\left\|x_{l}^{n+1}(t)-x_{l}^{n}(t)\right\| \leqslant & c_{1} \int_{0}^{t}\left\|x_{l}^{n+1}(\tau)-x_{l}^{n}(\tau)\right\| d \tau \\
& +c_{6} \int_{0}^{t}\left\|r^{n+1}(\tau)-r^{n}(\tau)\right\| d \tau \\
& +\int_{0}^{t}\left\|d^{n+1}(\tau)-d^{n}(\tau)\right\| d \tau \\
\leqslant & c_{1}\left\|x_{l}^{n+1}-x_{l}^{n}\right\|_{\lambda_{1}} \int_{0}^{t} e^{\lambda \tau} d \tau \\
& +c_{6}\left\|r^{n+1}-r^{n}\right\|_{\lambda_{2}} \int_{0}^{t} e^{\lambda(T-\tau)} d \tau \\
& +\left\|d^{n+1}-d^{n}\right\|_{\lambda_{1}} \int_{0}^{t} e^{\lambda \tau} d \tau .
\end{aligned}
$$

Multiplying both sides of the last expression by $e^{-\lambda t}$ and taking the supremum over $[0, T]$ on both sides yields

$$
\left\|x_{l}^{n+1}-x_{l}^{n}\right\|_{\lambda_{1}} \leqslant \underbrace{\frac{\lambda^{-1}}{1-\lambda^{-1} c_{1}}}_{m_{6}}\left\|d^{n+1}-d^{n}\right\|_{\lambda_{1}}+\underbrace{\frac{c_{6} c_{8}}{1-\lambda^{-1} c_{1}}}_{m_{7}}\left\|r^{n+1}-r^{n}\right\|_{\lambda_{2}} .
$$

Finally, the difference $x_{h}^{n+1}-x_{h}^{n}$ is bounded as follows on the basis of Equations 1 and 5,

$$
\begin{aligned}
x_{h}^{n+1}(t)-x_{h}^{n}(t)= & \int_{0}^{t}\left[f\left(x_{h}^{n+1}(\tau), u^{n+1}(\tau)\right)-f\left(x_{h}^{n}(\tau), u^{n}(\tau)\right)\right] d \tau, \\
\left\|x_{h}^{n+1}(t)-x_{h}^{n}(t)\right\| \leqslant & L_{1} \int_{0}^{t}\left\|x_{h}^{n+1}(\tau)-x_{h}^{n}(\tau)\right\| d \tau+L_{2} \int_{0}^{t}\left\|u^{n+1}(\tau)-u^{n}(\tau)\right\| d \tau \\
\leqslant & L_{1} \int_{0}^{t}\left\|x_{h}^{n+1}(\tau)-x_{h}^{n}(\tau)\right\| d \tau+c_{4} L_{2} \int_{0}^{t}\left\|x_{l}^{n+1}(\tau)-x_{l}^{n}(\tau)\right\| d \tau \\
& +c_{5} L_{2} \int_{0}^{t}\left\|r^{n+1}(\tau)-r^{n}(\tau)\right\| d \tau \\
\leqslant & L_{1}\left\|x_{h}^{n+1}-x_{h}^{n}\right\|_{\lambda_{1}} \int_{0}^{t} e^{\lambda \tau} d \tau+c_{4} L_{2}\left\|x_{l}^{n+1}-x_{l}^{n}\right\|_{\lambda_{1}} \int_{0}^{t} e^{\lambda \tau} d \tau \\
& +c_{5} L_{2}\left\|r^{n+1}-r^{n}\right\|_{\lambda_{2}} \int_{0}^{t} e^{\lambda(T-\tau)} d \tau .
\end{aligned}
$$


Multiplying the last expression by $e^{-\lambda t}$ and taking the supremum over $[0, T]$ on both sides yields

$$
\begin{aligned}
\left\|x_{h}^{n+1}-x_{h}^{n}\right\|_{\lambda_{1}} \leqslant & \underbrace{\frac{c_{5} c_{8} L_{2}}{1-\lambda^{-1} L_{1}}}_{m_{8}}\left\|r^{n+1}-r^{n}\right\|_{\lambda_{2}} \\
& +\underbrace{\frac{\lambda^{-1} c_{4} L_{2}}{1-\lambda^{-1} L_{1}}}_{m_{9}}\left\|x_{l}^{n+1}-x_{l}^{n}\right\|_{\lambda_{1}} .
\end{aligned}
$$

Using Equations A10 to A12, Equation A9 can be written in the same form as Equation 19,

$$
\left\|d^{n+1}-d^{n}\right\|_{\lambda_{1}} \leqslant \alpha\left\|d^{n}-d^{n-1}\right\|_{\lambda_{1}},
$$

where

$$
\begin{aligned}
\alpha= & m_{1}+m_{2} m_{5}+m_{3}\left(m_{6}+m_{5} m_{7}\right) \\
& +m_{4}\left(m_{5} m_{8}+\left(m_{6}+m_{5} m_{7}\right) m_{9}\right) .
\end{aligned}
$$

\section{APPENDIX B: PROOF OF PROPOSITION 1}

Using Equation A13, the following inequality is obtained for $m, n \in \mathbb{N}$ and $m>n$,

$$
\begin{aligned}
\left\|d^{m}-d^{n}\right\|_{\lambda_{1}} \leqslant & \left\|d^{m}-d^{m-1}\right\|_{\lambda_{1}}+\left\|d^{m-1}-d^{m-2}\right\|_{\lambda_{1}} \\
& +\ldots+\left\|d^{n+1}-d^{n}\right\|_{\lambda_{1}} \\
\leqslant & \alpha^{m-1}\left\|d^{1}-d^{0}\right\|_{\lambda_{1}}+\alpha^{m-2}\left\|d^{1}-d^{0}\right\|_{\lambda_{1}} \\
& +\ldots+\alpha^{n}\left\|d^{1}-d^{0}\right\|_{\lambda_{1}} \\
= & \left\|d^{1}-d^{0}\right\|_{\lambda_{1}} \alpha^{n} \sum_{k=0}^{m-n-1} \alpha^{k} \\
\leqslant & \left\|d^{1}-d^{0}\right\|_{\lambda_{1}} \alpha^{n} \sum_{k=0}^{\infty} \alpha^{k} \\
= & \left\|d^{1}-d^{0}\right\|_{\lambda_{1}} \frac{\alpha^{n}}{(1-\alpha)} .
\end{aligned}
$$

If $\alpha \in(0,1)$, then for all $\epsilon>0$, there exists $N \in \mathbb{N}$ such that for all $m, n>N,\left\|d^{m}-d^{n}\right\|_{\lambda_{1}}<\epsilon$, where $N \in \mathbb{N}$ can be chosen such that $\alpha^{N}<\frac{\epsilon(1-\alpha)}{\left\|d^{1}-d^{0}\right\|_{\lambda_{1}}}$. This shows that $\left\{d^{n}\right\}_{n=0}^{\infty}$ is a uniformly Cauchy sequence of continuous functions in $C^{0}([0, T])$, which is a complete normed linear space under $\|\cdot\|_{\lambda_{1}}$. Hence, $\lim _{n \rightarrow \infty} d^{n}=d^{\infty}$ for some $d^{\infty}$ uniformly on $[0, T]$.

Using Equations A10, A11, and A12, it follows similarly that $\left\{r^{n}\right\}_{n=0}^{\infty}\left\{x_{l}^{n}\right\}_{n=0}^{\infty}$ and $\left\{x_{h}^{n}\right\}_{n=0}^{\infty}$ are uniformly Cauchy sequence of continuous functions in $C^{0}([0, T])$, and hence $\lim _{n \rightarrow \infty} r^{n}=r^{\infty}, \lim _{n \rightarrow \infty} x_{l}^{n}=x_{l}^{\infty}$ and $\lim _{n \rightarrow \infty} x_{h}^{n}=x_{h}^{\infty}$ uniformly on $[0, T]$ for appropriate continuous functions $r^{\infty}, x_{l}^{\infty}$ and $x_{h}^{\infty}$, respectively. As an example, for $r^{n}$, we have

$$
\begin{aligned}
\left\|r^{m}-r^{n}\right\|_{\lambda_{2}} \leqslant & \left\|r^{m}-r^{m-1}\right\|_{\lambda_{2}}+\left\|r^{m-1}-r^{m-2}\right\|_{\lambda_{2}} \\
& +\ldots+\left\|r^{n+1}-r^{n}\right\|_{\lambda_{2}} \\
\leqslant & m_{5}\left(\left\|d^{m}-d^{m-1}\right\|_{\lambda_{1}}+\left\|d^{m-1}-d^{m-2}\right\|_{\lambda_{1}}\right. \\
& \left.+\ldots+\left\|d^{n+1}-d^{n}\right\|_{\lambda_{1}}\right) \\
\leqslant & \frac{m_{5}}{1-\alpha}\left(\alpha^{m-1}\left\|d^{1}-d^{0}\right\|_{\lambda_{1}}+\alpha^{m-2}\left\|d^{1}-d^{0}\right\|_{\lambda_{1}}\right. \\
& \left.+\ldots+\alpha^{n}\left\|d^{1}-d^{0}\right\|_{\lambda_{1}}\right) \\
= & \left\|d^{1}-d^{0}\right\|_{\lambda_{1}} \frac{m_{5} \alpha^{n}}{1-\alpha} \sum_{k=0}^{m-n-1} \alpha^{k} \\
\leqslant & \left\|d^{1}-d^{0}\right\|_{\lambda_{1}} \frac{m_{5} \alpha^{n}}{1-\alpha} \sum_{k=0}^{\infty} \alpha^{k} \\
= & \left\|d^{1}-d^{0}\right\|_{\lambda_{1}} \frac{m_{5} \alpha^{n}}{(1-\alpha)^{2}},
\end{aligned}
$$

where $m, n \in \mathbb{N}$ and $m>n$. Now, if $\alpha \in(0,1)$, then for all $\epsilon>0$, there exists $N \in \mathbb{N}$ such that for all $m, n>N$, it implies $\left\|r^{m}-r^{n}\right\|_{\lambda_{2}}<\epsilon$, where $N \in \mathbb{N}$ can be chosen such that $\alpha^{N}<\frac{\epsilon(1-\alpha)^{2}}{\left\|d^{1}-d^{0}\right\|_{\lambda_{1}} m_{5}}$.

From Equation 8,

$$
\left\|\dot{e}^{n}\right\|_{\lambda_{1}}=\frac{\left\|d^{n}-d^{n-1}\right\|_{\lambda_{1}}}{|k|},
$$

and it follows that $\lim _{n \rightarrow \infty}\left\|\dot{e}^{n}\right\|_{\infty}=0$. Since $e^{n}(0)=0$,

$$
\left\|e^{n}\right\|_{\infty}=\sup _{t \in[0, T]} \int_{0}^{t} \dot{e}^{n}(\tau) d \tau \leqslant\left\|\dot{e}^{n}\right\|_{\infty} T .
$$

Thus

$$
\lim _{n \rightarrow \infty}\left\|e^{n}\right\|_{\infty}=\lim _{n \rightarrow \infty}\left\|x_{h}^{n}-x_{l}^{n}\right\|_{\infty}=0,
$$

and $x_{h}^{\infty}=x_{l}^{\infty}$.

Using uniform convergence of sequences and passing to the limit, $J_{l}^{\infty}=\lim _{n \rightarrow \infty} J_{l}\left(x_{l}^{n}, u^{n}\right)=J_{l}\left(\lim _{n \rightarrow \infty} x_{l}^{n}, \lim _{n \rightarrow \infty} u^{n}\right)=$ $J_{l}\left(x_{l}^{\infty}, u^{\infty}\right)=J_{h}\left(x_{h}^{\infty}, u^{\infty}\right)=J_{h}\left(\lim _{n \rightarrow \infty} x_{h}^{n}, \lim _{n \rightarrow \infty} u^{n}\right)=$ $\lim _{n \rightarrow \infty} J_{h}\left(x_{h}^{n}, u^{n}\right)=J_{h}^{\infty}$. This completes the proof.

\section{APPENDIX C: COMPUTATION OF EQUATION 20}

We have

$$
\begin{aligned}
K_{1} & =R^{-1} B^{T} P=0.05 P, \\
K_{2} & =R^{-1} B^{T}=0.05, \\
\tilde{\mathrm{A}} & =\left(A-B K_{1}\right)=-0.1-0.0025 P, \\
\tilde{B} & =P .
\end{aligned}
$$

The $P$ in Equation 6 satisfies

$$
\begin{aligned}
\dot{P} & =0.2 P+0.0025 P^{2}-1, \\
P(T) & =10 .
\end{aligned}
$$


We can express $P$ as an explicit function of $t$ by separation of variables,

$$
P(t)=\frac{40 \sqrt{5}}{1-\exp \left(\frac{\sqrt{5}}{10}(t-2)+\ln \left(\frac{50-20 \sqrt{5}}{50+20 \sqrt{5}}\right)\right)}-40-20 \sqrt{5},
$$

and it is an increasing function of $t \in[0, T]$.

According to Equations A1 to A8,

$$
\begin{aligned}
& c_{1}=\sup _{t \in[0, T]}\|\tilde{\mathrm{A}}(t)\|=\sup _{t \in[0, T]}|0.1+0.0025 P(t)|=0.125, \\
& c_{2}=\sup _{t \in[0, T]}\left\|\tilde{\mathrm{A}}^{T}(t)\right\|=\sup _{t \in[0, T]}|0.1+0.0025 P(t)|=0.125, \\
& c_{3}=\sup _{t \in[0, T]}\|\tilde{B}(t)\|=\sup _{t \in[0, T]}|P(t)|=10, \\
& c_{4}=\sup _{t \in[0, T]}\left\|K_{1}(t)\right\|=\sup _{t \in[0, T]}|0.05 P(t)|=0.5, \\
& c_{5}=\left\|K_{2}\right\|=0.05, \\
& c_{6}=\left\|B K_{2}\right\|=0.0025, \\
& c_{7}=\sup _{t \in[0, T]} e^{-\lambda(T-t)} \int_{t}^{T} e^{\lambda \tau} d \tau=1.3834, \\
& c_{8}=\sup _{t \in[0, T]} e^{-\lambda t} \int_{0}^{t} e^{\lambda(T-\tau)} d \tau=1.3834 .
\end{aligned}
$$

Then, according to Equations A9 to A12,

$$
\begin{aligned}
& m_{1}=|1-k|=0.9, \\
& m_{2}=|k| e^{\lambda T}\left(c_{6}+c_{5} L_{2}\right)=0.0017 \\
& m_{3}=|k|\left(c_{1}+c_{4} L_{2}\right)=0.015, \\
& m_{4}=|k| L_{1}=0.01 \\
& m_{5}=\frac{c_{3} c_{7}}{1-\lambda^{-1} c_{2}}=17.4742 \\
& m_{6}=\frac{\lambda^{-1}}{1-\lambda^{-1} c_{1}}=2.1053 \\
& m_{7}=\frac{c_{6} c_{8}}{1-\lambda^{-1} c_{1}}=0.0044 \\
& m_{8}=\frac{c_{5} c_{8} L_{2}}{1-\lambda^{-1} L_{1}}=0.0042 \\
& m_{9}=\frac{\lambda^{-1} c_{4} L_{2}}{1-\lambda^{-1} L_{1}}=0.05
\end{aligned}
$$

where $L_{1}=0.1$ and $L_{2}=0.05$ are the Lipschitz constants of the high-fidelity model. Finally,

$$
\begin{aligned}
\alpha= & m_{1}+m_{2} m_{5}+m_{3}\left(m_{6}+m_{5} m_{7}\right) \\
& +m_{4}\left(m_{5} m_{8}+\left(m_{6}+m_{5} m_{7}\right) m_{9}\right)=0.9635 .
\end{aligned}
$$

\section{APPENDIX D: PROOF OF PROPOSITION 2}

Let $u^{*}$ denote an optimal control for the high-fidelity model and $x^{*}$ denote the corresponding state trajectory. Let
$J^{*}=J\left(x^{*}, u^{*}\right)$ denote the corresponding cost where $J(x, u)$ is defined by Equation 3. Consider the system,

$$
\dot{x}=A x+B u+d^{*}(t)
$$

obtained by evaluating $d_{h}(x, t)$ of Equation 21 at $x^{*}$, ie, $d^{*}(t)=$ $d_{h}\left(x^{*}(t), t\right)$. Note that the control-state pair $\left(u^{*}, x^{*}\right)$ represents a trajectory of Equation D1, such that the integration of

$$
\dot{x}=A x+B u^{*}+d^{*}(t), \quad x(0)=x^{*}(0),
$$

recovers the optimal trajectory $x^{*}$ of the high-fidelity model. For the same cost function $J(x, u)$, system D1 has an optimal control-state pair $\left(u^{\circ}, x^{\circ}\right)$, satisfying

$$
\dot{x}^{\circ}=A x^{\circ}+B u^{\circ}+d^{*}(t)
$$

and let the corresponding cost be $J^{\circ}$. Since $\left(u^{\circ}, x^{\circ}\right)$ is optimal for Equation D1,

$$
J^{\circ} \leqslant J^{*}
$$

Suppose the IMTR iterations converge and denote the corresponding control-state pair by $(\tilde{u}, \tilde{x})$. Then,

$$
\dot{\tilde{x}}=A \tilde{x}+B \tilde{u}+d_{l}(t),
$$

where

$$
d_{l}(t)=d_{h}(\tilde{x}(t), t)
$$

By Equation 22, Equation D6, and the triangle inequality, we have

$$
\begin{aligned}
\left\|d^{*}(t)-d_{l}(t)\right\| & =\left\|d_{h}\left(x^{*}(t), t\right)-d_{h}(\tilde{x}(t), t)\right\| \\
& =\left\|d_{h}\left(x^{*}(t), t\right)-\bar{d}(t)+\bar{d}(t)-d_{h}(\tilde{x}(t), t)\right\| \\
& \leqslant\left\|d_{h}\left(x^{*}(t), t\right)-\bar{d}(t)\right\|+\left\|d_{h}(\tilde{x}(t), t)-\bar{d}(t)\right\| \\
& \leqslant 2 \epsilon .
\end{aligned}
$$

Note that,

$$
\begin{aligned}
u^{\circ} & =-R^{-1} B^{T} P(t) x^{\circ}+R^{-1} B^{T} r^{\circ}, \\
\tilde{u} & =-R^{-1} B^{T} P(t) \tilde{x}+R^{-1} B^{T} \tilde{r},
\end{aligned}
$$

where $P(t)$ comes from Equation 6 and is the same in both cases, and

$$
\begin{aligned}
\dot{r}^{\circ} & =\bar{A}(t) r^{\circ}+\bar{B}(t) d^{*}(t), \\
\dot{r} & =\bar{A}(t) \tilde{r}+\bar{B}(t) d_{l}(t),
\end{aligned}
$$

where $\bar{A}(t)=-\left(A-B R^{-1} B^{T} P(t)\right)^{T}$ and $\bar{B}(t)=P(t)$. We evaluate systems D3 and D5 with the feedback control D8, and we have 


$$
\begin{aligned}
\dot{x}^{\circ} & =\tilde{\mathrm{A}}(t) x^{\circ}+\tilde{B}(t) r^{\circ}+d^{*}(t), \\
\dot{\tilde{x}} & =\tilde{\mathrm{A}}(t) \tilde{x}+\tilde{B}(t) \tilde{r}+d_{l}(t),
\end{aligned}
$$

where $\tilde{\mathrm{A}}(t)=\left(A-B R^{-1} B^{T} P(t)\right)$ and $\tilde{B}(t)=B R^{-1} B^{T}$. Note that $x^{\circ}(0)=\tilde{x}(0)$ and $r^{\circ}(T)=\tilde{r}(T)$. Let $\Delta x=x^{\circ}-\tilde{x}, \Delta r=r^{\circ}-\tilde{r}$ and $\Delta d(t)=d^{*}(t)-d_{l}(t)$. Then,

$$
\left[\begin{array}{c}
\Delta \dot{x} \\
\Delta \dot{r}
\end{array}\right]=\left[\begin{array}{cc}
\tilde{\mathrm{A}}(t) & \tilde{B}(t) \\
0 & \bar{A}(t)
\end{array}\right]\left[\begin{array}{c}
\Delta x \\
\Delta r
\end{array}\right]+\left[\begin{array}{c}
I \\
\bar{B}(t)
\end{array}\right] \Delta d
$$

where the input $\Delta d$ is bounded by $\|\Delta d(t)\| \leqslant 2 \epsilon$ for all $t \in$ $[0, T], \Delta x(0)=0$ and $\Delta r(T)=0$. From Equation D9,

$$
\begin{aligned}
\Delta r(t) & =\Phi(t, T) \Delta r(T)+\int_{T}^{t} \Phi(t, \tau) \bar{B}(\tau) \Delta d(\tau) d \tau \\
& =\int_{T}^{t} \Phi(t, \tau) \bar{B}(\tau) \Delta d(\tau) d \tau,
\end{aligned}
$$

where $\Phi(t, \tau)$ is the state transition matrix associated with $\bar{A}(t)$. Hence where $\Psi(t, \tau)$ is the state transition matrix associated with $\tilde{\mathrm{A}}(t)$. Using Equation D10, it follows that

$$
\|\Delta x(t)\| \leqslant 2 \epsilon t C_{2}(t),
$$

where $C_{2}(t)=\|\Psi(t, \tau) \tilde{B}(\tau)\|_{\infty}\left\|(T-\tau) C_{1}(\tau)\right\|_{\infty}+\|\Psi(t, \tau)\|_{\infty}$ and $\|\cdot\|_{\infty}$ is the supremum of $\|\cdot\|$ over $\tau \in[0, t]$. Note that $C_{2}(t)$ can be chosen to be finite and independent of $\epsilon$. Thus, $\|\Delta x(t)\|$ is $O(\epsilon)$.

Similarly, from Equation D8,

$$
\|\Delta u(t)\| \leqslant C_{3}(t)\|\Delta x\|+C_{4}\|\Delta r\|,
$$

where

$$
\begin{aligned}
C_{3}(t) & =\left\|R^{-1} B^{T} P(t)\right\|, \\
C_{4} & =\left\|R^{-1} B^{T}\right\|,
\end{aligned}
$$

are independent of $\epsilon$ and are bounded.

Finally, the Taylor expansion of $\tilde{J}$ about the trajectory $\left(u^{\circ}, x^{\circ}\right)$ gives

$$
\begin{aligned}
\tilde{J} & =\frac{1}{2} \tilde{x}(T)^{T} K_{f} \tilde{x}(T)+\frac{1}{2} \int_{0}^{T}\left[\tilde{x}(t)^{T} Q \tilde{x}(t)+\tilde{u}(t)^{T} R \tilde{u}(t)\right] d t \\
& =J^{\circ}-x^{\circ}(T)^{T} K_{f} \Delta x(T)-\int_{0}^{T}\left[x^{\circ}(t)^{T} Q \Delta x(t)+u^{\circ}(t)^{T} R \Delta u(t)\right] d t+O\left(\epsilon^{2}\right) .
\end{aligned}
$$

$$
\begin{aligned}
\|\Delta r(t)\| & =\left\|\int_{T}^{t} \Phi(t, \tau) \bar{B}(\tau) \Delta d(\tau) d \tau\right\| \\
& \leqslant \int_{t}^{T}\|\Phi(t, \tau) \bar{B}(\tau) \Delta d(\tau)\| d \tau \\
& \leqslant 2 \epsilon(T-t) C_{1}(t),
\end{aligned}
$$

where $C_{1}(t)=\|\Phi(t, \tau) \bar{B}(\tau)\|_{\infty}$ and $\|\cdot\|_{\infty}$ is the supremum of $\|\cdot\|$ over $\tau \in[t, T]$. Note that $C_{1}(t)$ can be chosen to be finite and independent of $\epsilon$. Thus, $\|\Delta r(t)\|$ is $O(\epsilon)$.

Similarly, from Equation D9 and $\Delta x(0)=0$,

$$
\Delta x(t)=\int_{0}^{t} \Psi(t, \tau)(\tilde{B}(\tau) \Delta r(\tau)+\Delta d(\tau)) d \tau,
$$

Therefore, on the basis of Equations D10, D11, and D12, the difference between $J^{\circ}$ and $\tilde{J}$ is $O(\epsilon)$.

In particular, because $(\tilde{u}, \tilde{x})$ is a control-state pair of trajectories of the high-fidelity model while $\left(u^{*}, x^{*}\right)$ is the optimal control-state pair of trajectories,

$$
J^{*} \leqslant \tilde{J} .
$$

On the basis of Equations D4 and D13, we have

$$
J^{\circ} \leqslant J^{*} \leqslant \tilde{J},
$$

and thus

$$
\left|\tilde{J}-J^{*}\right| \leqslant\left|\tilde{J}-J^{\circ}\right| .
$$

Thus, $\left|\tilde{J}-J^{*}\right|$ is $O(\epsilon)$ and the proof is complete. 